\title{
Correlation between Hot Carrier Stress, Oxide Breakdown and Gate Leakage Current for Monitoring Plasma Processing Induced Damage on Gate Oxide
}

\author{
Jan Ackaert ${ }^{1}$, Zhichun Wang ${ }^{2}$, Eddy De Backer', Cora Salm ${ }^{2}$ \\ 'Alcatel Microelectronics Westerring 15 B-9700 Oudenaande Belgium E-mail: jan.ackeart@mie.alcatel.be \\ 2MESA + Research Institute / University of Twente Semiconductor Components Group \\ P.O. Box 217, 7500 AE Enschede The Netherlands E-mail: Z.Wang@el.utwente.nl
}

\begin{abstract}
In this paper, we compare the HC stress and oxide breakdown results with the fast and commonly used gate leakage current measurement.

A clear correlation is found between low levels of gate leakage and both $\mathrm{HC}$ degradation and oxide breakdown. We, for the first time, demonstrate that the value of the gate leakage current is not only a failure indicator in the region about $1 \mathrm{nA}$ but also a good indicator of the reliability of the devices in the region between $1 \mathrm{pA}$ and $1 \mathrm{nA}$. Thus, from the value of gate leakage current, one can estimate the reliability of the devices, saving precious measurement time.
\end{abstract}

\section{INTRODUCTION}

For very-large-scale-integration

(VLSI)

manufacturing of integrated circuits (ICs), the use of high-density plasma enhanced deposition and anisotropic etching techniques is inevitable. However, since 1980s [1]-[3], degradation of gate oxides in MOS devices due to the electrical charging induced by plasma processing has been observed and reported. A lot of effort has been spent to understand the phenomena [4][5] and to find the method to monitor the charging damage [6]-[8]. The measurement techniques include gate leakage, threshold voltage shift, charge-tobreakdown and hot carrier stress. The charge-tobreakdown and the hot-carrier stress measurements are accurate measures of the gate oxide integrity [9]. However, they are not often used in monitoring damage because they are very time consuming $[10]$.

In this paper, we compare the HC stress and oxide breakdown results with the simple and commonly used gate. leakage current measurement [11]. The observed good correlation suggests that gate leakage current can be used to monitor device reliability under plasma stress, thereby saving a lot of measurement time.

\section{EXPERIMENTAI}

The test structures have been subjected to a $0.35 \mu \mathrm{m}$ CMOS backend-of-line process. The test structures are small transistors with $\mathrm{W} \times \mathrm{L}=$ $0.35 \mu \mathrm{m} \times 2 \mu \mathrm{m}$. The gate oxide thickness is $7.5 \mathrm{~nm}$. The charging tester consists of a MOS transistor with a finger-shaped metal antenna connected to the gate on the first or the second metal level. The antennas are patterned with a medium density plasma system. Oxide is deposited on top of the antennas with a highdensity plasma (HDP) system. The antenna ratio (AR), which is defined as the ratio between the area of the antenna connected to the transistor and the active area of the latter are $1 \mathrm{~K}, 10 \mathrm{~K}$, and $100 \mathrm{~K}$. To investigate the impact of the electron shading effect, comb shaped antennas with spacing of $0.6 \mu$ and $5 \mu$ are compared. The protection diode is connected to gate at subsequent level, in order to protect the gate oxide from damage produced by plasma process steps following the patterning antenna.

Gate leakage $\left(\mathrm{I}_{\mathrm{gleak}}\right)$ of the charging testers was measured by applying a gate voltage of $\mathrm{V}_{\mathrm{f}}=$ $3.6 \mathrm{~V}$. A charging tester can be considered to fail when the $I_{g \text { leak }}$ measured through its gate oxide exceeds $0.1 \mathrm{nA}$, indicating that soft or hard breakdown occurred. The $0 . \ln \mathrm{A}$ is selected as failure criterion based on the leak current distribution, as shown in figure 1 . The devices with $I_{\text {gleak }}$ higher than $0 . \ln A$, diverge from the intrinsic slope, indicating extrinsic damage induced by plasma charging.

Special precautions in the measurement setup have to be taken to reach high enough accuracy with the low-level leakage measurements. 


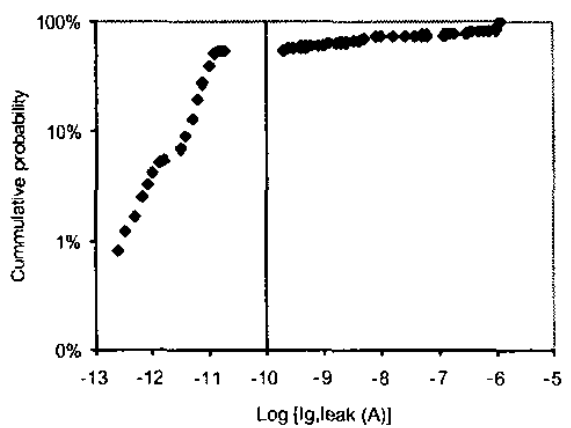

Figure 1. Distribution of gate leakage current. The devices with k,eak higher than $0 . \ln A$, diverge from the intrinsic slope, indicating extrinsic damage induced by plasma charging.

Hot carrier (HC) stress was also performed on pMOSFETs. The pMOSFET was $s$ tressed at $V_{d}=-$ $6.5 \mathrm{~V}, \mathrm{~V}_{\mathrm{g}}=-1.9 \mathrm{~V}$. This $\mathrm{V}_{\mathrm{g}}$ was chosen to maximize bulk current $\mathrm{b}$. The $\mathrm{HC}$ lifetime, which is defined as the time the MOSFET takes for a $10 \mathrm{mV}$ V shift, is compared with the initial fleak with $V_{z}=$ $3.6 \mathrm{~V}$ measured on the same devices.

The oxide breakdown measurement was performed with a ramping voltage stress on more than one thousand devices. The gate oxide was stressed on $3.5 \mathrm{~V}$, corresponding with $4.7 \mathrm{MV} / \mathrm{cm}$ for 1 second (step 1), $4.0 \mathrm{~V}$ or $5.3 \mathrm{MV} / \mathrm{cm}$ for 1 second (step 2), $4.5 \mathrm{~V}$ or $6.0 \mathrm{MV} / \mathrm{cm}$ for 1 second (step 3) and so on until $7.0 \mathrm{~V}$ or $9.3 \mathrm{MV} / \mathrm{cm}$ for 1 second (step 8). In between each step gate leakage is measured. The results are plotted as a function of the initial kleak with $\mathrm{V}_{\mathrm{g}}=3.6 \mathrm{~V}$. This testing method was developed to reveal latent as well as actual damage, for a wide range of gate oxide thickness in a very fast way.

\section{RESULTS}

The HC lifetime of pMOSFETs are shown in figure 2 for devices with metal 1 antennas and 3 for device with metal 2 antennas. For failing devices with $\mathrm{I}_{\text {,leak }}$ above $0.1 \mathrm{nA}$, the $\mathrm{HC}$ lifetime is degraded very much. The devices with $\mathrm{I}_{\mathrm{gleak}}$ in the range of 1 to $10 \mathrm{pA}$ have longer lifetimes, while the others with g,eak in the range of $10 \mathrm{pA}$ to $0.1 \mathrm{nA}$ value have clearly shorter lifetimes. A clear correlation is also observed with the size of the antenna connected to the gate of the transistor: increasing the antenna size by a decade, the $I_{g, \text { teak }}$ increases and the $\mathrm{HC}$ lifetime decreases by more than a decade. No impact of the spacing between the fingers is observed: in this case, electron shading is not the major cause of the degradation.



Figure 2. HC lifetime as function of gate leakage current measured at $3.6 \mathrm{~V}$ on the same devices with Metal 1 finger antenna. $S$ is the spacing between two fingers.

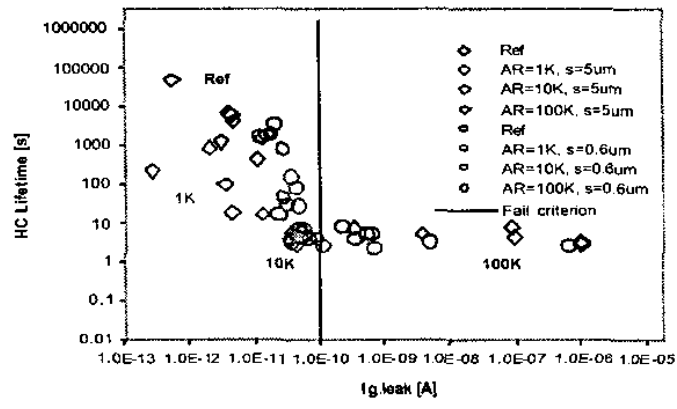

Figure 3. HC lifetime as function of gate leakage current measured at $3.6 \mathrm{~V}$ on the same devices with Metal 2 finger antenna. $S$ is the spacing between two fingers.

The strong correlation with the antenna ratio is considered as a signature for plasma damage being the cause of the degradation [12][13]. In this case the plasma damage can be caused by the etching of the metal pattem or/and the oxide deposition by a high density plasma process. Plasma damage during metal etching is ypically driven by electron shading and as such influenced by the spacing between the fingers of the antenna [14]-[17]. Since no impact is observed of the spacing between the fingers, it is more likely that the HC degradation is caused by the HDP oxide deposition. The mechanism and the required improvement on the HDP oxide deposition have been published before by the authors[18]. 
As well known, the HC mechanism of pMOSFET under $I_{b}$, max stress condition is the channel shortening effect due to electron trap near the drain region. This leads to increased drain current and reduced Vt. [19].

The almost 1:1 ratio between $\mathrm{HC}$ lifetime degradation and the antenna size is confinmed by other publications [20].

The results of breakdown measurement performed with a ramping voltage stress are compared with the gate leakage current on the same devices in figure 4 for devices with metal 1 antemnas and 5 for devices with metal 2 antennas.

All the reference structures without extra antenna connected to the gate have an initial leakage current in the range from $10 \mathrm{pA}$ to $0.1 \mathrm{nA}$ and break down after stress at $8 \mathrm{MV} / \mathrm{cm}$. The antenna devices that are as good as reference devices and with low initial bleak value also have breakdown values after stress at $8 \mathrm{MV} / \mathrm{cm}$, as shown in the figures. For the antenna devices with higher initial $\mathrm{I}_{\text {g,leak }}$ value, there is a clear linear trend between $I_{\text {g.jeak }}$ and the breakdown field.

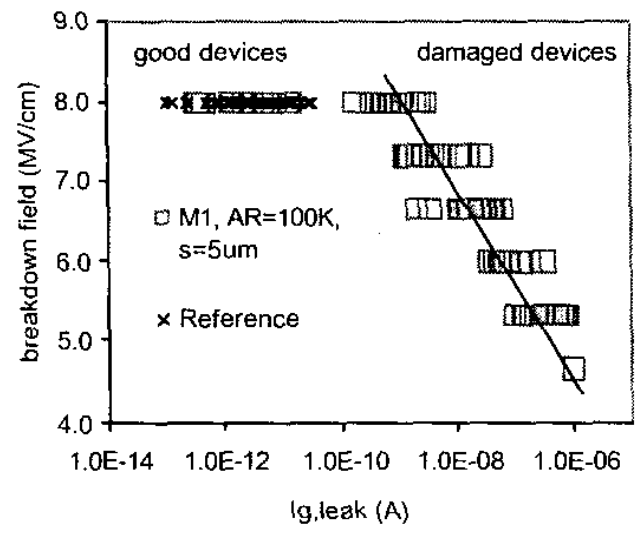

Figure 4. Breakdown field as function of initial gate leakage current measured on nMOSFETs with MI finger antenna.

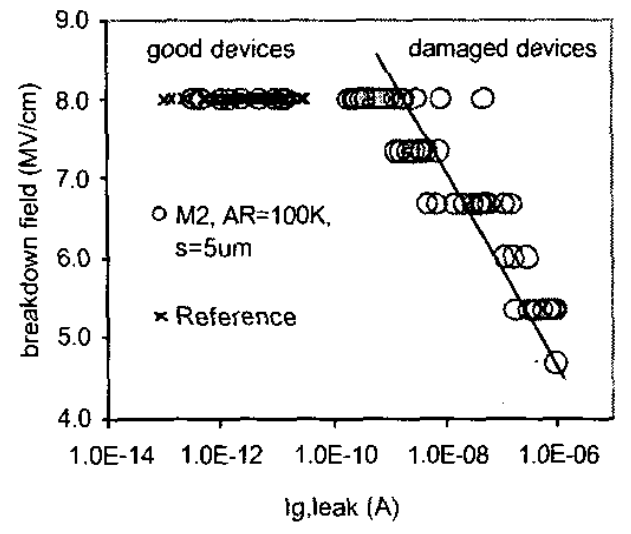

Figure 5. Breakdown field as function of initial gate leakage current measured on the same nMOSFETs with $M 2$ finger antenna.

\section{CONCLUSIONS}

A clear correlation is found between low levels of gate leakage and both $\mathrm{HC}$ degradation and oxide breakdown. We, for the first time, demonstrate that the value of the gate leakage current is not only a failure indicator in the region about $1 \mathrm{nA}$ but also a good indicator of the reliability of the devices in the region between $1 \mathrm{pA}$ and $1 \mathrm{nA}$. Thus, from the value of gate leakage current, one can estimate the reliability of the devices, saving precious measurement time.

\section{ACKNOWLEDGMENTS}

The authors are indebted to Geert van den Bosch and Wade Zawalsky of IMEC. Part of this work was funded by the Flemish Institute for Science and Technology (IWT) in project PINDA (Plasma Induced Damage), AUT/990241, in collaboration with IMEC, Belgium.

\section{REFERENCES}

[1]. T. Wadanabe and Y. Yoshida, "Dielectric breakdown of gate insulator due to reactive ion etching", Solid State Technology, P263, 1984.

[2]. K. Tsunokuni, K. Nojiri, S. Kuboshima and K. Hirobe, "The effect of charge build up on gate oxide breakdown during dry etching", Extended Abstract of Intemational Conference on Solid State Devices and Materials, p195, 1987.

[3]. F. Shone, K. Wu, J. Shaw, E. Hokelek, S. Mittal and A. Haranahalli, "Gate oxide charging and its elimination for metal antenna capacitor and transistor 
in VLSI CMOS double layer metal technology", Symposium on VLSI Technology, p73, 1989

[4]. H. Shin, D.King, T. Horiuchi, and C. Hu, "Thin oxide charging current during plasma etching of aluminum, IEEE Electron Device Letters, 12(8), p.404, 1991.

[5]. S. Fang, Murakawa S, Mcvittieand J. A new model for thin oxide degradation from wafer charging in plasma etching. International electron device meeting technical digest. 1992, p. 61.

[6]. H. Shin and C. Hu, "Monitoring plasmaprocess induced damage in thin oxide", IEEE Transaction on Semiconductor Manufacturing , 6(2), p.96, 1993.

[7]. S. Murakawa and J.P McVittie, "Direct measurement of surface charging during plasma etching", Part I. Japanese Journal of Applied Physics, 33(7B), p4446, 1994

[8]. C. T. Gabriel and J. L. Educate, "Application of Damage Measurement Techniques to a Study of Antenna Structure Charging", 2 $2^{\text {nd }}$ PPID, p91 -94, 1997.

[9]. B. Bhuva, P. Mongkolkachit, N. Bui, and S. Kerns, "A Study of Effects of Plasma-Induced Charging Damage on Hot-carrier Lifetime Using PreStressed Data", $4^{\text {th }}$ PPID, p69-72, 1999.

[10]. K. P. Cheung and E. J. Lloyd , "Fast Hot-Carrier Aging Method of Charging Damage Measurement", $4^{\text {th }}$ PPID, p208-211, 1999.

[11]. C. T. Gabriel and J. L. Educate, "Application of Damage Measurement Techniques to a Study of Antenna Structure Charging", $2^{\text {nd }}$ PPID, p91-94, 1997.

[12]. F. Shone, K. Wu, J. Shaw, E. Hokelek, S. Mittal and A. Haranahalli, "Gate oxide charging and its elimination for metal antenna capacitor and transistor in VLSI CMOS double layer metal technology", Symposium on VLSI Technology, p73, 1989.

[13]. H. Shin, C. C. King, T. Horiuchi, and C. Hu, "Thin Oxide Charging Current During Plasma Etching of Aluminum", ", IEEE Electron Device Letters, vol. 12, no. 8, p. 404-406, 1991.

[14]. K. Hashimoto, "New phenomena of charge damage in plasma etching: Heavy damage only trough denseline antenna", Jpn. J. Appl. Phys., vol 32, 1993, pp. 6109-6113.

[15]. K. Hashimoto, "Charge damage caused by electron shading effect", Jpn. J. Appl. Phys., vol 33, 1994, pp. 6013-6018.

[16]. A. Hasegawa, F. Shimpuku, M. Aoyama K. Hashimoto and M. Nakamura, "Direction of topography dependent damage current during plasma etching", Proc. $3^{\text {rd }}$ P2ID, p. 168, 1998.

[17]. M. Creusen, J. Ackaert, E. De Backer and G. Groeseneken, "Impact of reactor and transistor-type on electron shading effects," Proc. $4^{\text {th }}$ P2Id, pp. 8-11, 1999.

[18]. Jan Ackaert, Eddy De Backer, Peter Coppens, Martin Creusen "Prevention of plasma induced damage on thin gate oxide of HDP oxide deposition, metal etch, Ar preclean" Proc. $5^{\text {th }}$ P2Id, pp. 120-123,
2000. processing in BEOL sub-half micron CMOS processing.

[19]. Pei Yao "Hot-Carrier Degradation Study of High Density Plasma (HDP) Oxide Deposition Process in Deep-submicton NMOSFETS" SPIE 1999 Vol. 3884 pp. 116.123.

[20]. Sanjay Rangan "A Model for Channel Hot Carrier Reliability Degradation Due to Plasma Damage in MOS devices" 37 annual Intern. Reliability Physics Symposium San Diego 1999. 